\title{
Modelling of micro- and nanodomain arrays recorded in ferroelectrics-semiconductors by using atomic force microscopy
}

\author{
A.N. Morozovska \\ V. Lashkaryov Institute of Semiconductor Physics, NAS of Ukraine \\ 41, prospect Nauky, 03028 Kyiv, Ukraine \\ E-mail:morozo@i.com.ua
}

\begin{abstract}
The thermodynamical theory of nanodomain tailoring in ferroelectricssemiconductors allowing for semiconducting properties, screening and size effects is presented. The obtained analytical results prove that domains appearance is similar to the first order phase transition and completely agree with experimentally observed threshold nanodomains recording in $\mathrm{Pb}(\mathrm{Zr}, \mathrm{Ti}) \mathrm{O}_{3}$ and $\mathrm{LiTaO}_{3}$ thin films. The realistic dependence of equilibrium nanodomain radius over applied voltage in $\mathrm{BaTiO}_{3}, \mathrm{LiNbO}_{3}$ ferroelectricsemiconductors, $\mathrm{LiTaO}_{3}$ and $\mathrm{Pb}(\mathrm{Zr}, \mathrm{Ti}) \mathrm{O}_{3}$ thin films have been calculated. These results will help researchers both to achieve the reliable understanding of physical process taking place during nanoscale polarization reversal in the ferroelectric-semiconducting media and to determine the necessary conditions in order to record stable nanodomains with optimum lateral sizes and configuration to increase recording density and create various profiled microstructures.
\end{abstract}

Keywords: ferroelectric nanodomains, screening effects, atomic force microscopy, information recording.

Manuscript received 13.12.05; accepted for publication 29.03.06.

\section{Introduction}

Ferroelectric micro- and nanodomains (submicron spatial regions with reversed spontaneous polarization) can be created by inhomogeneous electric and/or elastic fields with definite polarity in many ferroelectricssemiconductors and photorefractive ferroelectrics [1-3]. Recently, one and two dimensional arrays of stable nanodomains have been tailored in $\mathrm{LiNbO}_{3}[4,5]$, $\mathrm{LiTaO}_{3}$ [6], $\mathrm{Pb}(\mathrm{Zr}, \mathrm{Ti}) \mathrm{O}_{3}$ [7-10], $\mathrm{BaTiO}_{3}$ [11] and $\mathrm{RbTiOPO}_{4}$ [12] ferroelectric materials using the electric fields caused by an atomic force microscope (AFM). The experimental and theoretical study of submicronand nanodomain tailoring in a ferroelectric media attract the broad interest owing to its possible applications in many fields of nanophysics and nanotechnology, namely: various nanostructures assembling for modern electronic and electromechanical devices [13], nonlinear and fine optical elements $[4,12]$, high density memory elements based on new physical principles [6, 7, 14]. From the fundamental viewpoint, formation of artificial nanodomains is a promising field for the direct observation of size, surface and interface effects of the domain structure and switching kinetics $[9,10]$.

However, in order to achieve these purposes it is necessary to develop an adequate physical model of the spontaneous polarization local reversal. Such models can help not only to optimize the problem of nanodomain writing with a high density and stability, but also to achieve a satisfactory understanding of complex physical processes taking place during the nanoscale polarization reversal.

However, existing phenomenological models give an incomplete picture of the nanodomain formation owing to the following reasons.

- Landauer [15] considered the domains nucleation during the polarization reversal in the homogeneous electric field. In this model, surface bond charges are compensated by the free charges on the flat metallic electrodes.

- Molotskii [16] evolved the model of domain formation inside the perfect ferroelectric-dielectric with free surface under the inhomogeneous electric field of AFM tip. However, he considered the depolarization field in the framework of the Landauer model as if the perfect external screening expected, although a nanosized AFM tip apex could be hardly treated as the plain electrode.

- In our recent papers [3, 17-19], we have tried to overcome the aforementioned discrepancies taking into consideration screening layers of immovable surface charges and semiconductor properties revealed by the most of ferroelectrics (see [2, 20-22]). In the most of 
experiments [4-12], the screening layer recharging is quite possible due to the field emission caused by the positively charged AFM tip [18, 19].

- Nanodomain tailoring in the thin ferroelectric film seems more useful for applications than domain recording in a relatively thick layer, allowing for possible miniaturization of functional elements. As far as we know, a theoretical description of nanodomain recording in thin ferroelectric films is in the initial stage; in particular, semiconductor properties of films are neglected (see e.g. [23-25]).

\section{Theoretical consideration of domain origin in ferroelectrics-semiconductors}

Let us consider a solid ferroelectric with spontaneous polarization $\mathbf{P}_{S}$, dielectric permittivity $\varepsilon_{i}$, electrical induction $\mathbf{D}=\varepsilon_{i} \cdot \mathbf{E}+4 \pi \mathbf{P}_{S}$, electric field $\mathbf{E}=-\nabla \varphi(\mathbf{r})(\varphi(\mathbf{r})$ is the electrostatic potential). We choose the spontaneous polarization $+P_{S}$ inside and $-P_{S}$ outside the domain (see Fig. 1). The AFM tip electric field is calculated in the spherical model, in which the charged sphere with radius $R_{0}$ substitutes the real tip. The validity of such approach for dielectric sample and other models are discussed in [26]. The voltage $U$ is applied between the tip and bottom electrode.

Surface charges with $\sigma_{S}=-P_{S}$ were captured on the sluggish trap levels before the domain formation
[27]. Usually $\Delta R<1 \mathrm{~nm}$ [4-12], thus, field emission is quite possible at applied voltages $U \geq U_{m}$. Keeping in mind the approach proposed in [28] for current-voltage characteristics of ferroelectric tunnel junctions as well as interface screening model evolved in [29], we assume that the switching current $J_{e} \sim \exp \left(-U_{m} / U\right)$. At applied voltages $U \geq U_{m}$, the amount of emitted carriers are quite enough to completely screen the reversed polarization of the domain butt-end [24, 30], i.e., $\sigma_{S} \rightarrow+P_{S}$ at $U>U_{m}$. Thus, interpolation for the equilibrium surface charge density $\sigma_{S}$ has the form:

$\sigma_{S}=\left\{\begin{array}{l}-P_{S}+2 P_{S} \exp \left(-U_{m} / U\right), \quad \sqrt{x^{2}+y^{2}}<d \\ -P_{S}, \quad \sqrt{x^{2}+y^{2}}>d\end{array}\right.$

In a general case, the activation voltage $U_{m}$ in some complex manner depends on the distance $\Delta R$, sample thickness $h$, etc. For the thin film, we assume that $U_{m} \cong E_{m} h$, where $E_{m}$ is the so-called activation field [1].

Another important experimental fact should be taken into consideration for the correct theoretical description of nanodomain tailoring using AFM tip. Namely, it seems quite probable that on the polar oxide surface thin water layers are quite possible $[8,25,31]$. Moreover, if AFM tip is wettable, its apex with curvature $25-50 \mathrm{~nm}$ can be completely covered with

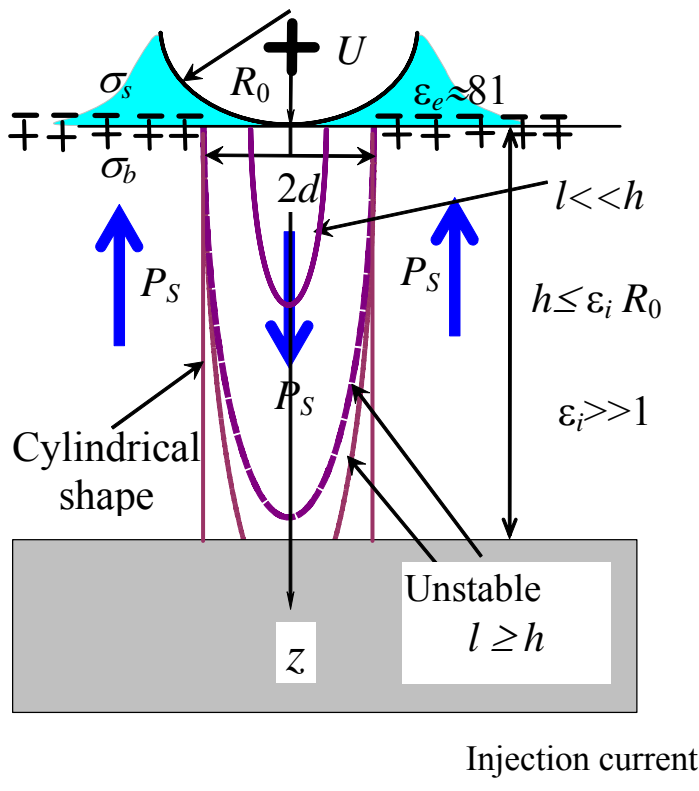

2D-array of domains

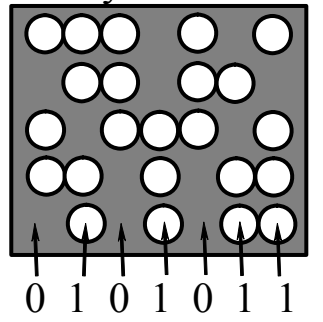

AFM tip apex

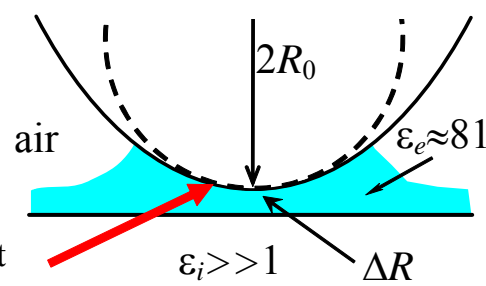

Fig. 1. Nanodomain growth through the thin ferroelectric film. $U$ is the applied voltage, $\Delta R$ is the distance between the tip apex and sample surface, $R_{0}$ is the tip radius of curvature, $d$ is the domain radius, $l$ is the domain length, $h$ is the film thickness, $P_{S}$ is the spontaneous polarization, $\sigma_{S}$ is the density of surface charges captured on the sluggish trap levels, $\sigma_{b}$ is the density of bound charges related to $P_{S}$ discontinuity. 
water [32]. Hereinafter, we regard that this region has the effective dielectric permittivity $\varepsilon_{e}$.

Electric field screening is realized by free charges with the bulk density $\rho_{f}(\mathbf{r}) \approx-\varepsilon_{i}(\mathbf{r}) / 4 \pi R_{d}^{2} \quad\left(R_{d}\right.$ is the Debye screening radius). The electrostatic potential spatial distribution should be determined from the Maxwell equation $\varepsilon_{i} \Delta \varphi(\mathbf{r})=-4 \pi \rho_{f}(\mathbf{r})$ supplemented with the interfacial conditions $D_{n \text { int }}=D_{n \text { ext }}$ on the domain surface $\Sigma, D_{n \text { int }}-D_{n \text { ext }}=4 \pi \sigma_{S}$ on the free surface $z=0$. Thus, we obtain the following boundary problem:

$$
\begin{aligned}
& \Delta \varphi_{0}(\mathbf{r})=0, \quad z \leq 0, \\
& \left.\varphi_{0}\right|_{\mathbf{r} \in \operatorname{tip}}=U, \quad \varphi_{0}(z=0)=\varphi(z=0), \\
& \left.\left(\varepsilon_{e} \frac{\partial \varphi_{0}}{\partial z}-\varepsilon_{i} \frac{\partial \varphi}{\partial z}\right)\right|_{z=0}=\left\{\begin{array}{cc}
4 \pi\left(\sigma_{S}-P_{S}\right), & \sqrt{x^{2}+y^{2}}<d, \\
0, & \sqrt{x^{2}+y^{2}}>d,
\end{array}\right.
\end{aligned}
$$$$
\Delta \varphi(\mathbf{r})-\frac{\varphi(\mathbf{r})}{R_{d}^{2}}=0, \quad z \geq 0,
$$$$
\left.\varepsilon_{i}\left(\frac{\partial \varphi_{\text {int }}}{\partial n}-\frac{\partial \varphi_{\text {ext }}}{\partial n}\right)\right|_{\Sigma}=\left.8 \pi\left(\mathbf{P}_{S} \mathbf{n}\right)\right|_{\Sigma}, \quad \varphi(z=h)=0 .
$$

In order to apply all the following results to the anisotropic semiconductor, one can make the substitution $\quad z \rightarrow z \sqrt{\varepsilon_{a} / \varepsilon_{c}}, \quad l \rightarrow l \sqrt{\varepsilon_{a} / \varepsilon_{c}}$, $\varepsilon_{i} \rightarrow \sqrt{\varepsilon_{c} \varepsilon_{a}}, R_{d}^{2}=\frac{\varepsilon_{a} k_{\mathrm{B}} T}{4 \pi e^{2} n_{d}}$. Here $\varepsilon_{a}$ and $\varepsilon_{c}$ are anisotropic dielectric permittivity values perpendicular and parallel to the polar axis $z$.

Our calculations $[18,19]$ have shown that in the thick films with the thickness $h \gg>R_{d}$ (when $\left.\exp \left(-h / R_{d}\right)<<1\right)$ the equilibrium domain length $l$ almost always is much greater than its radius $d$, i.e., usually semi-ellipsoidal domains are spike-like. In contrast to the thick films, equilibrium domains usually grow through the thin film with the thickness

$$
\begin{aligned}
& \Phi_{U}(d)= \\
& = \begin{cases}\frac{\sqrt{\varepsilon_{a} \varepsilon_{c}}+\varepsilon_{e}}{\sqrt{\varepsilon_{a} \varepsilon_{c}}-\varepsilon_{e}} \ln \left(\frac{\sqrt{\varepsilon_{a} \varepsilon_{c}}+\varepsilon_{e}}{2 \varepsilon_{e}}\right) \times \frac{4 \pi \varepsilon_{e}\left(\sigma_{S}-P_{S}\right) \cdot U R_{0} R_{d}\left(\sqrt{R_{0}^{2}+d^{2}}-R_{0}\right)}{\left(\sqrt{\varepsilon_{a} \varepsilon_{c}}+\varepsilon_{e}\right) R_{d}+2 \sqrt{\varepsilon_{a} \varepsilon_{c}} \sqrt{R_{0}^{2}+d^{2}}}, & h>>R_{d} \\
\frac{\sqrt{\varepsilon_{a} \varepsilon_{c}}+\varepsilon_{e}}{\sqrt{\varepsilon_{a} \varepsilon_{c}}-\varepsilon_{e}} \ln \left(\frac{\sqrt{\varepsilon_{a} \varepsilon_{c}}+\varepsilon_{e}}{2 \varepsilon_{e}}\right) \times \frac{4 \pi \varepsilon_{e}\left(\sigma_{S}-P_{S}\right) \cdot U R_{0} \cdot h R_{d}\left(\sqrt{R_{0}^{2}+d^{2}}-R_{0}\right)}{\left(\sqrt{\varepsilon_{a} \varepsilon_{c}}+\varepsilon_{e}\right) h R_{d}+\sqrt{\varepsilon_{a} \varepsilon_{c}}\left(R_{d}+2 h\right) \sqrt{R_{0}^{2}+d^{2}}}, & h \leq \sqrt{\varepsilon_{c} \varepsilon_{a}} R_{0} .\end{cases}
\end{aligned}
$$


At $R_{d} \rightarrow \infty, h \rightarrow \infty$ and $\sigma_{S} \rightarrow-P_{S}, \varepsilon_{e}=1$ the energy (5) coincides with the one calculated in [16].

c) The depolarization field energy $\Phi_{D}(d, l)=\Phi_{D L}(d, l)+\Phi_{D S}(d)$ is caused by local polarization reversal. The Landauer energy $\Phi_{D L}(d, l)$ is absent for the cylindrical domain that reached the bottom electrode. Pade approximation for the Landauer energy of the prolate semi-ellipsoidal domain acquires the form $[18,19]$ :

$$
\begin{aligned}
& \Phi_{D L}(d, l) \approx \\
& \approx\left\{\begin{aligned}
\frac{16 \pi^{2} P_{S}^{2}}{\sqrt{\varepsilon_{a} \varepsilon_{c}}} \frac{d^{4} R_{d}\left(\ln \left(2 \sqrt{\varepsilon_{a} / \varepsilon_{c}} \cdot l / d\right)-1\right)}{3 \sqrt{\varepsilon_{a} / \varepsilon_{c}} l R_{d}+4 d^{2}\left(\ln \left(2 \sqrt{\varepsilon_{a} / \varepsilon_{c}} \cdot l / d\right)-1\right)}, \\
d<<l \\
h>R_{d} \\
0, \quad h \leq \sqrt{\varepsilon_{c} \varepsilon_{a}} \cdot R_{0} .
\end{aligned}\right.
\end{aligned}
$$

The energy (6) coincides with that calculated in [16] at $R_{d} \rightarrow \infty$. The domain butt energy $\Phi_{D S}(d)$ has the form [19]:

$$
\begin{aligned}
& \Phi_{D S}(d) \approx \\
& \approx\left\{\begin{array}{c}
\frac{\pi^{2}\left(\sigma_{S}-P_{S}\right)^{2} d^{3} R_{d}}{\sqrt{\varepsilon_{a} \varepsilon_{c}} d+\left(\sqrt{\varepsilon_{a} \varepsilon_{c}}+\varepsilon_{e}\right) 3 \pi R_{d} / 16}, \\
d \widetilde{>} R_{0} / 2, \quad h>>R_{d}, \\
\frac{\pi^{2}\left(\sigma_{S}-P_{S}\right)^{2} d^{3} h R_{d}}{h\left(\sqrt{\varepsilon_{a} \varepsilon_{c}} d+\left(\sqrt{\varepsilon_{a} \varepsilon_{c}}+\varepsilon_{e}\right) 3 \pi R_{d} / 16\right)+\sqrt{\varepsilon_{a} \varepsilon_{c}} R_{d} d / 2} \\
h \leq \sqrt{\varepsilon_{c} \varepsilon_{a}} R_{0} .
\end{array}\right.
\end{aligned}
$$

It should be noted that the similar problem of domain intergrowth through the thin ferroelectricdielectric film was considered by Molotskii [25]. However, the depolarization field energy dependence on the film thickness and domain radius was chosen as $d^{2} h$ under the condition $h \gg d$. As it follows from our energy (7), the dependence $d^{2} h$ can be expected in the opposite case $h<<d$ [19].

The free energy (3) dependence on the domain radius for various values of the applied voltage is represented in Fig. 2. It is clear from Fig. 2 that the free energy has no minimum at small voltages. With the voltage increase, the absolute minimum $\Phi_{\min }<0$ appears at a critical value of the domain radius $d_{\min }$. Under the further voltage increase, the radius of the stable domain increases.

It is clear from Fig. 2 that there is a potential barrier between $d=0$ (domain is absent) and $d \neq 0$ (domain exists). The relative height of this barrier decreases with

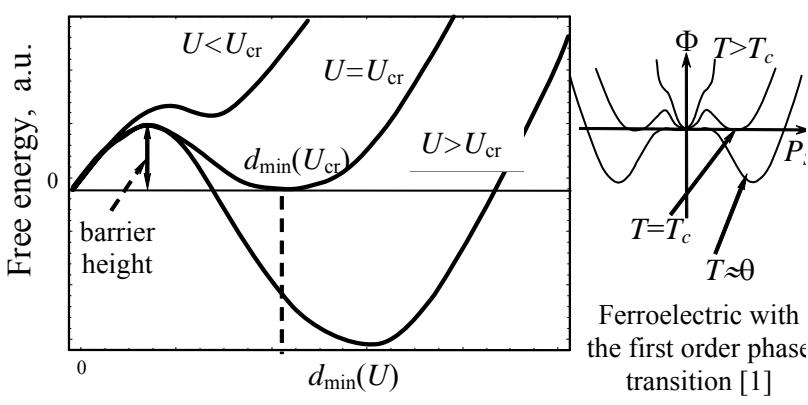

Domain radius $d$, a.u.

Fig. 2. Schematic free energy dependence on the domain radius $d$ for the prolate semi-ellipsoidal or cylindrical inter-grown domains at different applied voltage values $U$ (left plot). Compare it with a typical free energy dependence on polarization $P_{S}$ at different temperatures $T$ for ferroelectric with the first order phase transition (right plot).

applied voltage increase. That is why, the domain radius does not decrease continuously with voltage decrease: the stable domain appears with a non-zero radius $d=d_{\min }$ at $U \geq U_{\mathrm{cr}}$, i.e., when homogeneous polarization distribution becomes unstable. Such "threshold" domain formation is similar to the wellknown first order phase transition. At lower voltages $U<U_{\mathrm{cr}}$, domain formation becomes energetically impossible. This result seems to be quite reasonable, because the most of stable ferroelectric nanodomains were recorded only above some critical voltage $U_{\mathrm{cr}} \sim(3 \ldots 6) \mathrm{V}$ (see e.g. [6-8]).

From the condition $\Phi_{\min }\left(d_{\min }, U\right)=0$, we derived the following expressions for $U_{\mathrm{cr}}$ and $d_{\min }\left(U_{\mathrm{cr}}\right)$ determination valid for nanodomain recording in the thin film with $h<<R_{d}, h \leq \sqrt{\varepsilon_{c} \varepsilon_{a}} R_{0}$ :

$$
\begin{aligned}
& U_{\mathrm{cr}}(h) \approx \sqrt{\frac{32 \psi_{S}}{3}} \frac{\sqrt{\varepsilon_{a} \varepsilon_{c}} R_{0}+\left(\sqrt{\varepsilon_{a} \varepsilon_{c}}+\varepsilon_{e}\right) h}{\sqrt{\left(\sqrt{\varepsilon_{a} \varepsilon_{c}}+\varepsilon_{e}\right) h}} \times \\
& \times\left(\varepsilon_{e} \frac{\sqrt{\varepsilon_{a} \varepsilon_{c}}+\varepsilon_{e}}{\sqrt{\varepsilon_{a} \varepsilon_{c}}+\varepsilon_{e}} \ln \left(\frac{\sqrt{\varepsilon_{a} \varepsilon_{c}}+\varepsilon_{e}}{2 \varepsilon_{e}}\right)\right)^{-1}, \\
& d_{\min }\left(U_{\mathrm{cr}}\right) \approx \sqrt{\frac{3 \psi_{S}\left(\sqrt{\varepsilon_{a} \varepsilon_{c}}+\varepsilon_{e}\right)}{8\left(\sigma_{S}\left(U_{\mathrm{cr}}\right)-P_{S}\right)^{2}}} h .
\end{aligned}
$$

It is clear from (9) that in order to record domains with a minimum radius, it is necessary to choose the ferroelectric medium with high spontaneous polarization $P_{S}$, small domain wall energy $\psi_{S}$ and permittivity $\sqrt{\varepsilon_{a} \varepsilon_{c}}$, but not very small Debye screening radius (namely it should satisfy the conditions 
$\left.R_{d}>>h, \quad R_{d}>>R_{0}\right)$. Also, it seems urgent to decrease the film thickness $h$ until ferroelectric phase exists $[1,41,42]$, but keeping in mind that $\left(\sigma_{S}\left(U_{\mathrm{cr}}\right)-P_{S}\right) \sim 2 P_{S}\left(\exp \left(-U_{m} / U_{\mathrm{cr}}\right)\right)-1$ accordance with (1) and $U_{m} \cong E_{m} h$, the film thickness $h$ should not be extremely small in order to prevent sharp increase of $d_{\min }\left(U_{\mathrm{cr}}\right)$ (see denominator in (9)). Taking into account that the threshold field $E_{m}, \psi_{S}$, and $R_{d}$ could not be directly calculated within the framework of our model, they should be determined as fitting parameters from the experimental dependence $d(U)$. When material parameters $E_{m}, \psi_{S}, R_{d}$ are known and regarded almost independent on the film thickness $h$, as well as the possible thickness dependence $\varepsilon_{a, c}(h)$ is determined [41], an optimum $h$ value can be calculated numerically from the condition $d_{\min }\left(U_{\mathrm{cr}}(h)\right)=\min$. For the thickness-independent permittivity $\varepsilon_{a, c}$, we derived the approximation:

$h_{\mathrm{opt}} \approx \frac{\left(32 \psi_{S} / 3\right)^{1 / 3}}{\sqrt{\varepsilon_{a} \varepsilon_{c}}+\varepsilon_{e}}\left(\frac{\sqrt{\varepsilon_{a} \varepsilon_{c}}\left(\sqrt{\varepsilon_{a} \varepsilon_{c}}-\varepsilon_{e}\right)}{\varepsilon_{e} \ln \left(\sqrt{\varepsilon_{a} \varepsilon_{c}}+\varepsilon_{e} / 2 \varepsilon_{e}\right)} \frac{2 R_{0}}{E_{m}}\right)^{2 / 3}$.

Allowing for the fact that it is much more difficult to change the material parameters $E_{m}, \psi_{S}$, and $R_{d}$ than the film thickness $h$, the proposed procedure for the optimum thickness determination seems necessary.

For the majority of experiments [4-7], recorded nanodomains remain unchangeable for many days and weeks. This fact proves their stability and is extremely useful for many applications. It has the following explanation within the framework of the proposed model. When the applied voltage $U$ is turned off, the external field disappears in proportion to $U$, and the domain depolarization field $E_{D}=-\nabla \varphi_{D} \sim\left(\sigma_{S}-P_{S}\right)$ vanishes due to the surface traps sluggish recharging (i.e., when $\sigma_{S} \rightarrow P_{S}$ ). The remained positive surface energy $\Phi_{C}(d) \sim \psi_{S} d h$ tends to reverse the domain. In order to move, the domain wall has to overcome the pinning caused by defects, but the tension field $E_{W} \cong \psi_{S} / P_{S} d \sim 100 \mathrm{kV} / \mathrm{cm}$ appeared too small in comparison with the thermodynamic coercive field $E_{C} \cong 4 \pi P_{S} / \sqrt{\varepsilon_{a} \varepsilon_{c}} \sim(250 \ldots 2700) \mathrm{kV} / \mathrm{cm} \quad$ regarded necessary for the domain repolarization [8]. Thus, the domain wall pinning does provide the recorded domains stability; surely it also determines the domain growth kinetics [7, 10].

Let us discuss the peculiarities of possible interinfluence between domains during their recording. Neighbour nanodomains can interact via their depolarization fields. If the time interval $t$ between the neighbour domains recording is sufficient for complete recharging the surface traps, depolarization field vanishes and domains inter-influence is absent. Thus, during such process it is possible to obtain independent stable nanodomains with the minimum radius $d_{\min }$ at $U \approx U_{\mathrm{cr}}$ (see (8) and (9)).

\section{Modelling of stable ferroelectric domains}

\subsection{Screening and size effects on the nanodomains recording in $\mathrm{BaTiO}_{3}$}

It should be noted that Eng et al. [11] recorded nanodomains with the radius about $30 \mathrm{~nm}$ in $\mathrm{BaTiO}_{3}$ crystals at the applied voltage $80 \mathrm{~V}$. The dependence of the equilibrium domain radius $d$ over the applied voltage $U$ is shown in Fig. 3 for various Debye screening radii $R_{d}$.

We would like to underline that Debye screening not only decreases depolarization field inside the domain but also shields the AFM tip electric field inside the sample. As a result, the Debye screening radius decrease leads to the decrease of the equilibrium domain radius (compare the lowest curves with the dashed ones). The critical voltage $U_{\mathrm{cr}}$ is almost independent over $R_{d}$ value at small radii of curvature $R_{0}<<R_{d}$ and distance $\Delta R<<R_{d} / \sqrt{\varepsilon_{a} \varepsilon_{c}}$, e.g. $U_{\mathrm{cr}} \approx 2.5 \mathrm{~V}$ corresponds to $R_{d} \geq 250 \mathrm{~nm}$ (see (8)).

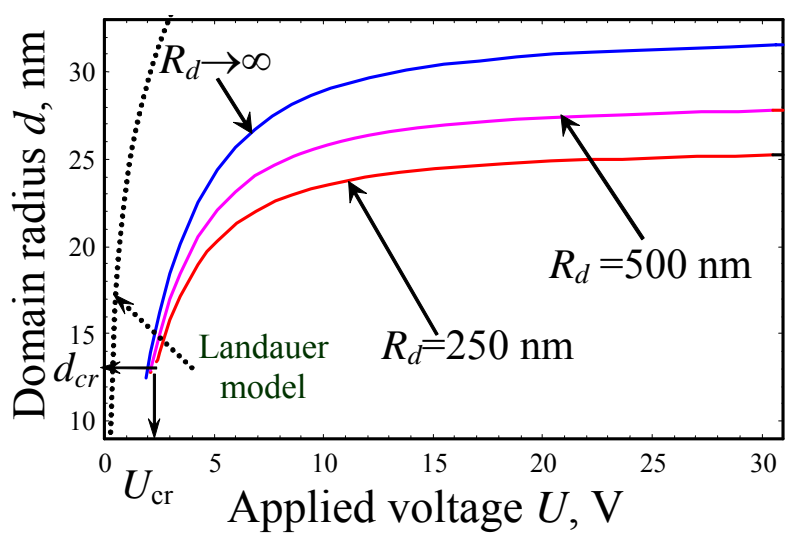

Fig. 3. Equilibrium domain radius $d$ dependence on the applied voltage $U$ for various Debye screening radii $R_{d}$ (solid curves). Dashed curves represent calculations without domain butt depolarization energy and screening effects (Landauer model [15]). We assume that $\varepsilon_{e}=81, \Delta R \leq 1 \mathrm{~nm}, R_{0}=25 \mathrm{~nm}, U_{m}=$ $=1.25 \mathrm{~V}$ and $h \gg R_{d}$. Other parameters are typical for $\mathrm{BaTiO}_{3}$ [17]: $P_{S} \approx 26 \mu \mathrm{C} / \mathrm{cm}^{2}, \psi_{S} \approx 8 \mathrm{~mJ} / \mathrm{m}^{2}$ [36], $\varepsilon_{a}=2000, \varepsilon_{c}=120$. 
In a general case, carriers emission leads to the essential decrease of domain sizes at high voltages, namely, the domain growth stops at $U>>U_{m}$ (see saturated curves for $U>20 \mathrm{~V}$ ). Indeed, $\sigma_{S} \rightarrow P_{S}$ at $U_{m} \rightarrow 0$, and so emitted carriers fully compensate not only the depolarization field caused by the domain butt polarization, but they simultaneously screen the charged tip electric field, which is the reason of domain formation (see (5) and (7)).

Note that the domain butt depolarization field energy (7) essentially decreases the domain sizes even at low voltages [17]. It does not appear in the system considered in [16] due to the complete screening of surface bond charges by the free charge inside the upper electrode (see dotted curves).

In the following section, we'll show that our free energy (3) can be unambiguously reconstructed using experimental dependence $d(U)$, i.e., how the parameters $\psi_{S}, R_{d}$ and $U_{m}$ can be determined. The procedure is rather simple. First, the dependence $d(U)$ should be calculated from (3) and compared with equilibrium domain sizes recorded at definite $h, R_{0}$ and $\Delta R$ values. The values $\varepsilon_{a}, \varepsilon_{c}, \varepsilon_{e}$ and $P_{S}$ are regarded known or measured independently for the chosen pair film-substrate. The values $\psi_{S}, \quad R_{d}$ and $U_{m}$ should be determined as fitting parameters from the dependence $d(U)$.

\subsection{Submicrodomain recording in $\mathrm{LiNbO}_{3}$}

Let us apply our theoretical results to domain formation in $\mathrm{LiNbO}_{3}$ single crystals using high-voltage AFM. In experiments $[4,5]$, the AFM tip radius was $R_{0}=50 \mathrm{~nm}$, distance $\Delta R<1 \mathrm{~nm}$, maximum applied voltage pulse value $U_{\text {max }}=4 \mathrm{kV}$ with duration up to 5 min, sample thickness $h \sim 1 \mathrm{~mm}$. For $\mathrm{LiNbO}_{3}$ at room temperature, $\varepsilon_{a}=84$, $\varepsilon_{C}=30, P_{S} \approx 50 \mu \mathrm{C} / \mathrm{cm}^{2}, \quad \psi_{S} \approx(5 . .50) \mathrm{mJ} / \mathrm{m}^{2} \quad$ (see e.g. [40]). The comparison of experimental results and our calculations is presented in the Fig. 4 . The fitting value of the Debye screening radius $R_{d}>100 \mu \mathrm{m}$ is in a reasonable agreement with estimations $R_{d} \sim$ $\left(10^{4} \ldots 10^{6}\right) \mathrm{nm}$ valid for the broad gap ferroelectricssemiconductors with unavoidable growth defects.

\subsection{Nanodomain recording in $\mathrm{Pb}(\mathrm{Zr}, \mathrm{Ti}) \mathrm{O}_{3}$ and $\mathrm{LiTaO}_{3}$ thin films}

Recently, nanodomain arrays were recorded in $\mathrm{Pb}(\mathrm{Zr}, \mathrm{Ti}) \mathrm{O}_{3}$ thin films using AFM. In the experiment [7], the AFM tip radius $R_{0}=(20 \ldots 50) \mathrm{nm}$, the distance $\Delta R \leq 0.3 \mathrm{~nm}$ was difficult to control due to the surface roughness close to $0.2 \ldots 0.3 \mathrm{~nm}$, applied voltage pulse values varied in the range $U=(4 \ldots 12) \mathrm{V}$ with the duration about $0.1 \mathrm{~s}$. The comparison of experimental results and our calculations is presented in Fig. 5.

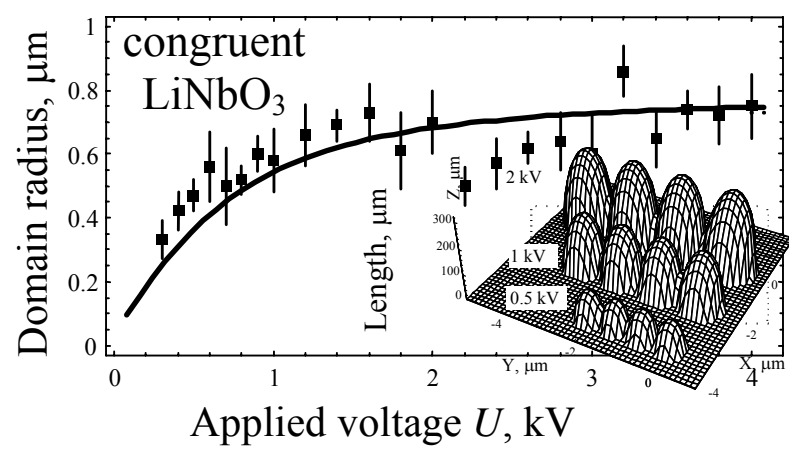

Fig. 4. Equilibrium domain radius and length vs the applied voltage $U$ in $\mathrm{LiNbO}_{3}$. Squares with error bars are experimental data from [4] for the domain radius $d$, the domain length was estimated as 150 to $250 \mu \mathrm{m}$ [5], $R_{0}=50 \mathrm{~nm}, \Delta R<$ $<1 \mathrm{~nm}$. The solid curve is our fitting at $R_{d} \geq 100 \mu \mathrm{m}, \psi_{S} \approx$ $\approx 5 \mathrm{~mJ} / \mathrm{m}^{2}$ [40], $U_{m}=0.15 \mathrm{kV}, P_{S} \approx 50 \mu \mathrm{C} / \mathrm{cm}^{2}, \varepsilon_{a}=85, \varepsilon_{c}=$ $=30, \varepsilon_{e}=81$. Inset: $3 \mathrm{D}$ view of nanodomains arrays recorded in congruent $\mathrm{LiNbO}_{3}$ sample at variuos voltages $U$.

Reconstruction of the free energy dependence on the domain radius is performed for several values of the applied voltage $U$.

The spontaneous polarization $P_{S}$ and dielectric permittivity components $\varepsilon_{a, c}$ were calculated using coefficients [43] for the bulk $\mathrm{PbZr}_{0.2} \mathrm{Ti}_{0.8} \mathrm{O}_{3}$. It appeared that the value $P_{S} \approx 70 \mu \mathrm{C} / \mathrm{cm}^{2}$ coincides with that extracted from the $a / c$ ratio measured in the same thin films [37]. The fitting value of the surface energy $\psi_{S} \approx 140 \mathrm{~mJ} / \mathrm{m}^{2}$ is of the same order as the $\mathrm{PbTiO}_{3}$ domain wall energy $(100 \ldots 200) \mathrm{mJ} / \mathrm{m}^{2}$ calculated by $[34,35]$ from the first principles. Thus, our fitting makes it possible to reconstruct the surface free energy value in $\mathrm{Pb}(\mathrm{Zr}, \mathrm{Ti}) \mathrm{O}_{3}$ thin film.

It is clear from Fig. 5 that the free energy has no minimum at the small voltages $U \leq 4 \mathrm{~V}$. With the voltage increase above $U \leq 6 \mathrm{~V}$, the absolute minimum $\Phi_{\text {min }}(d)<0$ appears at the definite value of the domain radius $d \approx 25 \mathrm{~nm}$. With the further voltage increase, the equilibrium domain radius rapidly increases and the stable domain appears (see curves for $U \geq 8 \mathrm{~V}$ ).

There is a potential barrier between $d=0$ (domain is absent) and $d \neq 0$ (domain exists). The relative height of this barrier sharply increases with the applied voltage decrease (compare curves for $U=6 \mathrm{~V}$ and $U=15 \mathrm{~V}$ ). That is why, the domain radius does not decrease continuously with the voltage decrease: the domain appears with the non-zero radius $d_{\min }=\left(U_{\mathrm{cr}}\right)$ at $U>U_{\mathrm{cr}}$. It is seen from the figure that the critical voltage $U_{\mathrm{cr}} \approx 5.7 \mathrm{~V}$, which is in a good agreement with the value reported by Paruch et al. [7]. 


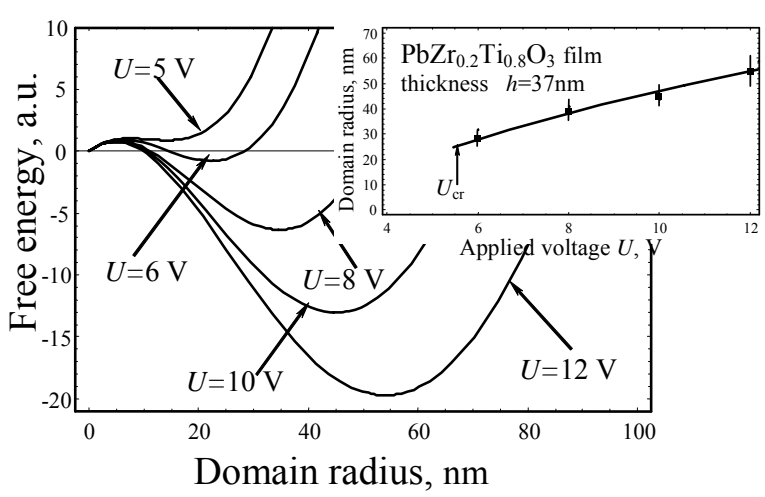

Fig. 5. Free energy dependence on the nanodomain radius and applied voltage (basic plot) was reconstructed from the dependence $d(U)$ (inset). Squares with error bars are experimental data from [7] for nanodomain radius recorded in the $\mathrm{PbZr}_{0.2} \mathrm{Ti}_{0.8} \mathrm{O}_{3}$ thin film on $\mathrm{SrTiO}_{3}$ substrate with thickness $h=$ $=37 \mathrm{~nm}, \varepsilon_{a}=143, \varepsilon_{c}=86, \varepsilon_{e}=81, P_{S} \approx 70 \mu \mathrm{C} / \mathrm{cm}^{2}$ [43] and $R_{0}=(20 \ldots 50) \mathrm{nm}, \Delta R \leq 0.3 \mathrm{~nm}$. Solid line is our fitting obtained at $\psi_{\mathrm{S}} \approx 140 \mathrm{~mJ} / \mathrm{m}^{2}, R_{d}=1 \mu \mathrm{m}, R_{0}=35 \mathrm{~nm}$, activation voltage $U_{m} \approx 1.5 \mathrm{~V}, \Delta R \leq 0.5 \mathrm{~nm}$.

The optimization procedure based on (8)-(10) proves that it is possible to record stable domains with $d_{\text {min }} \approx 17 \mathrm{~nm}$ at $U_{\mathrm{cr}} \approx(6 \ldots 8) \mathrm{V}$ in the films with the thickness $h_{\mathrm{opt}} \approx(70 \ldots 130) \mathrm{nm}$. Thus, information recording with the density $86 \mathrm{Gbit} / \mathrm{cm}^{2}$ is accessible in the thin PZT films (compare with the density 4 to $28 \mathrm{Gbit} / \mathrm{cm}^{2}$ reported in $\left.[7,10]\right)$.

Note that the activation field fitting value $E_{m} \approx U_{m} / h=405 \mathrm{kV} / \mathrm{cm}$ is in a surprisingly good agreement with the quasi-static coercive field values $E_{c} \sim(40 \ldots 400) \mathrm{kV} / \mathrm{cm}$ typical for thin PZT films (see e.g. [20] and refs therein).

Let us apply our theoretical results (3) to (10) to the nanodomain recording in thin congruent $\mathrm{LiTaO}_{3}$ films using high-voltage AFM. Fujimoto et al. [6] used the AFM tip with the radius $R_{0}=25 \mathrm{~nm}$, the maximum applied voltage pulse value was $U_{\max }=11 \mathrm{~V}$ with duration up to $10^{-4} \mathrm{~s}$, and $\mathrm{LiTaO}_{3}$ thin films had the thickness $h=(55 \ldots 83) \mathrm{nm}$. The comparison of experimental results and our calculations is represented in Fig. 6.

The $\mathrm{LiTaO}_{3}$ domain wall surface energy density $\psi_{S}$ can vary within the range $(60 . .400) \mathrm{mJ} / \mathrm{m}^{2}$ (see [40] and refs therein). The spontaneous polarization value $P_{S} \approx 55 \mu \mathrm{C} / \mathrm{cm}^{2}$ was obtained in the same $\mathrm{LiTaO}_{3}$ samples at $1 \mathrm{kHz}$ [6]. Note that the fitting value of the carrier emission threshold field $E_{m} \approx U_{m} / h=$ $=1455 \mathrm{kV} / \mathrm{cm}$ is of the same order as the thermodynamic coercive field value of perfect $\mathrm{LiTaO}_{3}$. The relatively small Debye screening radius $R_{d}=100 \mu \mathrm{m}$ could be explained by the presence of numerous lithium vacancies reported by Fujimoto et al.

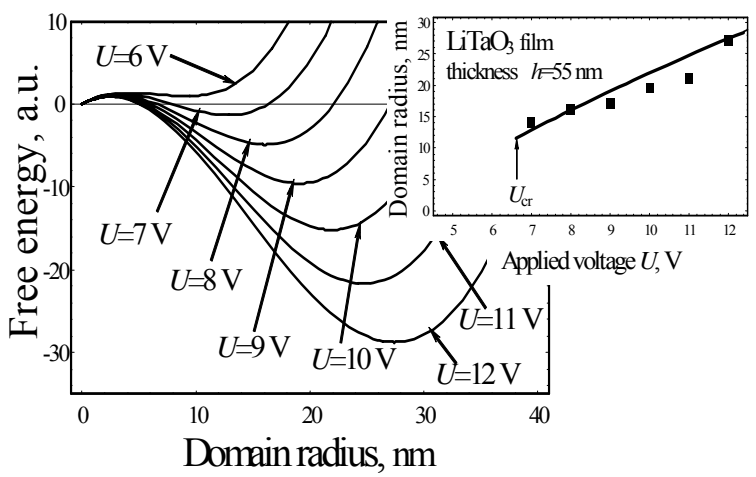

Fig. 6. Free energy dependence on the nanodomain radius (basic plot) reconstructed from the dependence $d(U)$ (inset). Squares are experimental data from [6] for the nanodomain radius recorded in the thin $\mathrm{LiTaO}_{3}$ film with the thickness $h=$ $=55 \mathrm{~nm}, R_{0}=25 \mathrm{~nm}$. Solid line is our fitting calculated at: $P_{S} \approx 55 \mu \mathrm{C} / \mathrm{cm}^{2}, \varepsilon_{a}=53, \varepsilon_{c}=45[40], \varepsilon_{e}=81, \psi_{S} \approx$ $220 \mathrm{~mJ} / \mathrm{m}^{2}, R_{d}=100 \mu \mathrm{m}, \Delta R \leq 1 \mathrm{~nm}, U_{m} \approx 6.5 \mathrm{~V}$.

[6] as well as by the carriers injection from the tip. But the question about the applicability of our "equilibrium" calculations remains open due to the small duration of the applied voltage pulses $\sim 10^{-4} \mathrm{~s}$. Despite this warning, we obtained rather well quantitative agreement between our fitting and experimental points.

The optimization procedure based on (8)-(10) gives that it is possible to record stable domains with $d_{\text {min }} \approx 8.5 \mathrm{~nm}$ at $U_{\mathrm{cr}} \approx 4.4 \mathrm{~V}$ in the films with the thickness $h_{\mathrm{opt}} \approx(20 \ldots 30) \mathrm{nm}$. Thus, information recording with the density $338 \mathrm{Gbit} / \mathrm{cm}^{2}$ is accessible in the thin $\mathrm{LiTaO}_{3}$ films.

\section{Conclusion}

The obtained results will help researchers to achieve the reliable understanding of physical process taking place during the micro- and nanoscale polarization reversal in the ferroelectric-semiconducting media, as well as to determine the necessary experimental conditions in order to record stable nanodomains with optimum lateral sizes and configuration in order to increase recording density and create different profiled microstructures. Namely:

- The thermodynamical theory of nanodomain tailoring in ferroelectrics-semiconductors has been evolved allowing for semiconducting properties, screening and size effects. The contribution of these effects leads to the significantly new description of the physical processes taking place under the nanoscale polarization reversal, in particular, they determine equilibrium nanodomain sizes.

- It has been demonstrated that the domain appearance is similar to the well-known first order phase transition: the domain appears with non-zero minimum radius at definite critical voltage applied to the AFM tip. 
The lateral sizes of nanodomains recorded at different voltages applied to the AFM tip were calculated in $\mathrm{BaTiO}_{3}, \quad \mathrm{LiNbO}_{3}$ ferroelectricsemiconductors and $\mathrm{Pb}(\mathrm{Zr}, \mathrm{Ti}) \mathrm{O}_{3}, \mathrm{LiTaO}_{3}$ thin films.

\section{References}

1. M.E. Lines, A.M. Glass, Principles and applications of ferroelectrics and related phenomena. Oxford University Press, Oxford, 1978.

2. V.M. Fridkin, Ferroelectrics semiconductors, Consultant Bureau, New-York and London, 1980.

3. A.N. Morozovska, E.A. Eliseev, V.V. Obukhovsky // Ferroelectrics 288, p. 265-275 (2003).

4. A. Agronin, Y. Rosenwaks, and G. Rosenman // Appl. Phys. Lett. 85, p. 452-454 (2004).

5. M. Molotskii, A. Agronin, P. Urenski, M. Shvebelman, Y. Rosenwaks and G. Rosenman // Phys. Rev. Lett. 90, 107601-4 (2003).

6. K. Fujimoto, Y. Cho // Appl. Phys. Lett. 83, p. 5265-5267 (2003).

7. P. Paruch, T. Tybell, J.-M. Triscone // Appl. Phys. Lett. 79, p. 530-532 (2001).

8. C. Durkan, M. E. Welland, D.P. Chu, and P. Migliorato // Phys. Rev. B 60, p. 16198-16204 (1999).

9. C.H. Ahn, K.M. Rabe, and J.-M. Triscone // Science 303, p. 488-490 (2004).

10. T. Tybell, P. Paruch, T. Giamarchi, and J.-M. Triscone // Phys. Rev. Lett. 89, 097601-4 (2002).

11. L.M. Eng, M. Bammerlin, Ch. Loppacher, M. Guggisberg, R. Bennewitz, R. Luthi, E. Meyer, Th. Huser, H. Heinzelmann and H.-J. Guntherodt // Ferroelectrics 222, p. 153-159 (1999).

12. Y. Rosenwaks, D. Dahan, M. Molotskii, and G. Rosenman // Appl. Phys. Lett. 86, 012909-3 (2005).

13. S.V. Kalinin, D.A. Bonnell, T. Alvarez, X. Lei, Z. $\mathrm{Hu}, \mathrm{R}$. Shao, and J.H. Ferris // Adv. Mater. 16, p. 795-799 (2004).

14. J.F. Scott, Ferroelectric memories. Springer, Berlin and Heidelberg, 2000.

15. R. Landauer // J. Appl. Phys., 28 p. 227-234 (1957).

16. M. Molotskii // J.Appl. Phys. 93 p. 6234-6237 (2003).

17. A.N. Morozovska, E.A. Eliseev // Phys. status solidi (b) 242, p. R79-R81 (2005).

18. A.N. Morozovska, E.A. Eliseev // E-print archive cond-mat http://arxiv.org/cond-mat/0508045 (2005).

19. A.N. Morozovska, E.A. Eliseev // E-print archive cond-mat http://arxiv.org/cond-mat/0509450 (2005).

20. A.N. Morozovska, E.A. Eliseev // J. Phys.: Condens. Matter. 16, p. 8937-8956 (2004).
21. A.N. Morozovska, E.A. Eliseev // Phys. status solidi (b) 242, p. 947-961 (2005).

22. A.N. Morozovska, E.A. Eliseev // Physica B, 355, p. 236-243 (2005).

23. X. Li, A. Mamchik, I.-W. Chen // Appl. Phys. Lett. 79, p. 809-811 (2001).

24. B. Wang, and C.H. Woo // J. Appl. Phys. 94, p. 4053-4059 (2003).

25. M. Molotskii // J. Appl. Phys. 97, p. 014109-8 (2005).

26. S.V. Kalinin, E. Karapetian, M. Kachanov // Phys. Rev. B 70 184101-24 (2004).

27. G.I. Rosenman, E.I. Boikova, Yu.L. Chepelev // Phys. status solidi (a) 69, p. K1 73 - K1 77 (1982).

28. H. Kohlstedt, N.A. Pertsev, J. Rodrigues Contreras, R. Waser // Phys. Rev. B 72, 125341-10 (2005).

29. M. Grossmann, O. Lonse, D. Bolten, U. Boettger, R. Waser // J. Appl. Phys. 92, p. 2680-2696 (2002).

30. T. Tybell, C.H. Ahn, J.-M. Triscone // Appl. Phys. Lett. 75, p. 856-858 (1999).

31. F. Felten, G.A.Schneider, J. Munoz Saldana, S.V. Kalinin // J. Appl. Phys. 96 p. 563-568 (2004).

32. J. Jang, G.C. Schatz, and M.A. Ratner // J. Chem. Phys. 120, p. 1157-1160 (2004).

33. R.C. Miller, G. Weinreich // Phys. Rev. 117, p. 1460-1466 (1960).

34. S. Poykko, D.J. Chadi // Appl. Phys. Lett. 75, p. 2830-2832 (1999).

35. B. Meyer, D. Vanderbilt // Phys. Rev. B 65, 104111-11 (2002).

36. J. Padilla, W. Zhong, D. Vanderbilt // Phys. Rev. B 53, p. R5969-R5973 (1996).

37. C. Lichtensteiger, J.-M. Triscone, J. Junquera, P. Ghosez // E-print archive cond-mat http://arxiv.org/cond-mat/0404228 (2004).

38. D.D. Fong, G.B. S.K. Stephenson, Streiffer, J.A. Eastman, O. Auciello, P.H. Fuoss, C. Thompson // Science 304, p. 1650-1653 (2004).

39. T.J. Yang, U. Mohideen // Physics Letters A, 250, p. 205-210 (1998).

40. D.A. Scrymgeour, V. Gopalan, A. Itagi, A. Saxena, and P.J. Swart // Phys. Rev. B 71, 184110-11 (2005).

41. M.D. Glinchuk, E.A. Eliseev, V.A. Stephanovich // Physica B 332, p. 356-370 (2002).

42. M.D. Glinchuk, A.N. Morozovska // J. Phys.: Condens. Matter. 16, p. 3517-3531 (2004).

43. L.E. Cross, Ferroelectric ceramics: Tailoring properties for specific application. In: Ferroelectric ceramics / Ed. N. Setter. Birkhauser Verlag, Basel, 1993. 